\title{
The GEYSERS Optical Testbed: a Platform for the Integration, Validation and Demonstration of Cloud-based Infrastructure Services
}

Bartosz Belter ${ }^{1}$, Juan Rodriguez Martinez ${ }^{2}$, José Ignacio Aznar ${ }^{2}$, Jordi Ferrer Riera ${ }^{3}$, Luis M. Contreras ${ }^{2}$, Monika Antoniak-Lewandowska ${ }^{4}$, Matteo Biancani ${ }^{5}$, Jens Buysse ${ }^{6}$, Chris Develder $^{6}$, Yuri Demchenko ${ }^{7}$, Pasquale Donadio $^{8}$, Dimitra Simeonidou ${ }^{9}$, Reza Nejabati ${ }^{9}$, Shuping Peng ${ }^{9}$, Łukasz Drzewiecki ${ }^{4}$, Eduard Escalona $^{3}$, Joan Antoni Garcia Espin ${ }^{3}$, Steluta Gheorghiu ${ }^{3}$, Mattijs Ghijsen ${ }^{7}$, Jakub Gutkowski ${ }^{1}$, Giada Landi $^{10}$, Gino Carrozzo ${ }^{10}$, Damian Parniewicz ${ }^{1}$, Philip Robinson ${ }^{11}$, Sebastien Soudan ${ }^{12}$

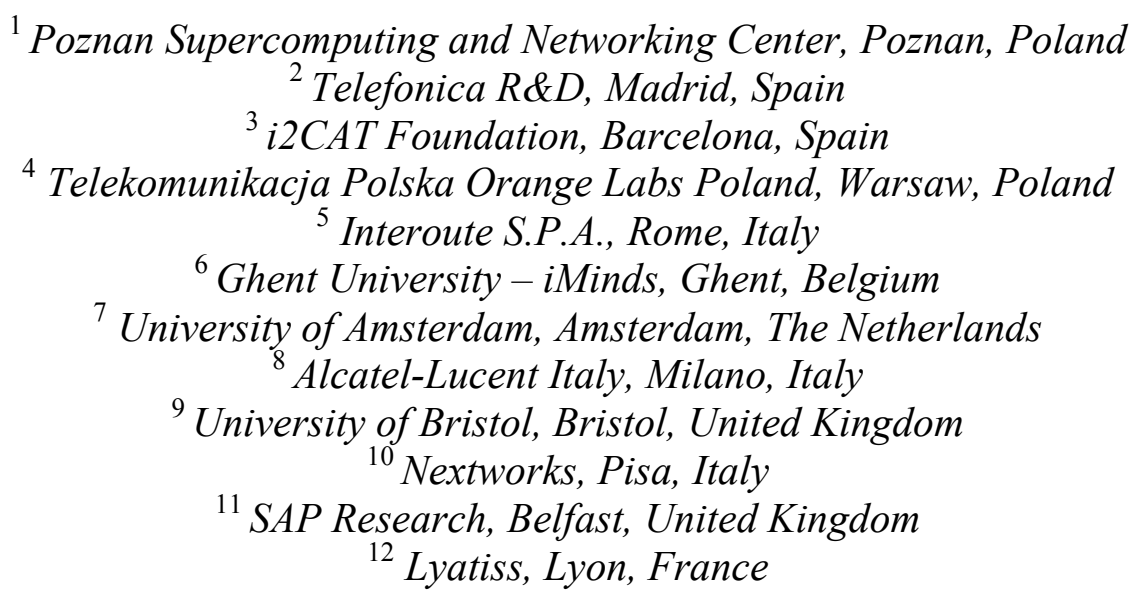

\begin{abstract}
The recent evolution of cloud services is leading to a new service transformation paradigm to accommodate network infrastructures in a cost-scalable way. In this transformation, the network constitutes the key to efficiently connect users to services and applications. In this paper we describe the deployment, validation and demonstration of the optical integrated testbed for the "GEneralized architecture for dYnamic infrastructure SERviceS" (GEYSERS) project to accommodate such cloud based Infrastructure Services. The GEYSERS testbed is composed of a set of local physical testbeds allocated in the facilities of the GEYSERS partners. It is built up based on the requirements specification, architecture definition and per-layer development that constitutes the whole GEYSERS ecosystem, and validates the procedures on the GEYSERS prototypes. The testbed includes optical devices (layer 1), switches (layer 2), and IT resources deployed in different local testbeds provided by the project partners and interconnected among them to compose the whole testbed layout. The main goal of the GEYSERS testbed is twofold. On one hand, it aims at providing a validation ground for the architecture, concepts and business models proposed by GEYSERS, sustained by two main paradigms: Infrastructure as a Service (IaaS) and the coupled provisioning of optical network and IT resources. On the other hand, it is used as a demonstration platform for testing the software prototypes within the project and to demonstrate to the research and business community the project approach and solutions. In this work, we discuss our experience in the deployment of the testbed and share the results and insights learned from our trials in the process. Additionally, the paper highlights the most relevant experiments carried out in the testbed, aimed at the validation of the overall GEYSERS architecture.
\end{abstract}

Keywords: Future Internet Architecture, Cloud Computing, Intercloud Architecture, Optical Testbeds, Validation, Infrastructure Services, Cloud, Infrastructure Virtualization 


\section{Introduction}

The current development of cloud computing technologies not only demonstrates an emerging trend towards the integration of cloud based infrastructure services through new inter-cloud and hybrid models, architectures and integration tools. It also supports the provisioning of a common/interoperable environment for migrating existing infrastructures and services to virtualised cloud-based architectures [1][2].

Enhanced Cloud Computing services such as Infrastructure as a Service (IaaS), Platform as a Service (PaaS) or Software as a Service (SaaS) require the dynamic allocation and virtualization of hardware and software elements in order to optimize resource utilization [3][4]. These requirements stretch the communication network and protocols, especially when cloud facilities (e.g., Data Centres (DCs)) are interconnected through the Internet. Moreover, there is a need to support these services with enhanced network resource provisioning mechanisms in a cost-effective scalable and dynamic way [5]. Finally, bandwidth requirements [9] and computing capacity for future applications will stress and exceed current network and IT infrastructure capabilities. Consequently, providers are facing the challenge to adapt their current infrastructure services provisioning models.

The ability to evolve both Cloud environments and IT and network resources to meet these challenges is crucial for a successful integration of cloud-based services and represents a current milestone in evolutional computing and communication technologies. This does not only pertain to technical issues, but also drives new business opportunities for Telecom providers. For instance, enterprise oriented cloud usage scenarios already demand combined computing and network resource provisioning [6]. This implies issues such as low latency, guaranteed bandwidth, application-centric management, security service consistency and energy efficiency [7]. The combined cloud and network resource provisioning requires that a number of services and control systems interoperate at different stages of the whole provisioning process [8].

In general, cloud based applications operate as regular applications, in particular using modern Service Oriented Architecture (SOA) Web Services platforms for services and applications integration. However, their composition and integration into distributed multi-domain, multi-provider heterogeneous cloud based infrastructure will require a number of new/additional functionalities, services and protocols to manage their provisioning and operation [10][11]. Development of such new functionalities motivates the creation of a new type of testbeds that would allow experimenting with the different components of inter-cloud infrastructures, integrating traditional and cloud based IT resources and providing controllable (and configurable) network services.

In this paper, we present a multi-domain testbed with the key target of providing a multipurpose environment for testing and validating cloud-oriented technologies for provisioning of complex infrastructure on-demand services, comprising cloud-based and traditional compute, storage and network resources. Additionally the testbed provides a reference model for the Cloud Carrier functional/operational model and cloud Network as a Service (NaaS) model, whose goal is to implement a manageable network infrastructure for inter-cloud integration as proposed in the NIST Cloud Computing Reference Architecture (CCRA) [1]. The GEYSERS testbed is mostly composed of optical equipment provided by the project partners, since the primary focus of the GEYSERS project research and development is on the service operation procedures for optical infrastructures. However, the adoption of the GEYSERS 
architecture is not limited to optical technologies; a wider evaluation of the GEYSERS architecture and the proposed solutions allows assuming that the architecture is capable of introducing any circuit-oriented networking technology. The work also presents the rationale for building the testbed and details capabilities of each local site, as a part of the whole validation environment. Finally, the paper also highlights the most relevant experiments carried out in the testbed, aimed at the validation of the overall GEYSERS architecture.

The remainder of the paper is organized as follows: Section 2 briefly presents the project's innovative architecture, built over three layers which lead to the newly defined business roles: owner of the physical infrastructure, owner of the virtual infrastructure and operator of the virtual infrastructure. Then, the global project testbed is described in Section 3. This testbed is constructed by interconnecting local testbeds provided by most of the project partners that are geographically distributed over seven European countries. GEYSERS procedures, experiments and demonstrators are described in Section 4, which provides a clear idea on the benefits that GEYSERS brings through different business models. The paper is concluded in Section 5.

\section{New Future Internet architectures - the GEYSERS approach}

The GEYSERS project proposes and explores a key innovative architecture for cloud-oriented virtual infrastructure provisioning, capable of: (i) seamless and coordinated provisioning of virtual infrastructures composed of network and IT resources, and (ii) the end-to-end network service delivery that overcomes limitations of the network/domain segmentation. This is achieved by the adoption of the concepts of IaaS and service oriented networking, enabling infrastructure operators to offer new converged network and IT services as part of the underlying IaaS cloud infrastructure. On the one hand, the service-oriented paradigm and IaaS framework enable flexibility of infrastructure provisioning in terms of configuration, accessibility and availability for the user. On the other hand, the layer-based structure of the GEYSERS architecture enables separation of functional aspects of each of the entities involved in the converged service provisioning, from the service consumer to the physical ICT infrastructure.

\subsection{An overview of the GEYSERS Architecture}

Figure 1 shows the layering structure of the GEYSERS architecture reference model. Each layer is responsible for the implementation of different functionalities covering full end-to-end service delivery from the service layer to the physical substrate. Central to the GEYSERS architecture and focus of the project are the enhanced Network Control Plane $(\mathrm{NCP}+)$, and the novel Logical Infrastructure Composition Layer (LICL). The Service Middleware Layer (SML) represents existing solutions for service management. At the lowest level, the Physical Infrastructure layer comprises network and IT resources from different Physical Infrastructure Providers. The different resources contained within the lowest level of the architecture depicted in Figure 1 consists of (i) optical network resources, both fiberand lambda-switching capable, located in the core networks, and (ii) IT resources dedicated to computing and storage tasks, located at the edges and interconnected among them through the high-capacity optical network devices. 


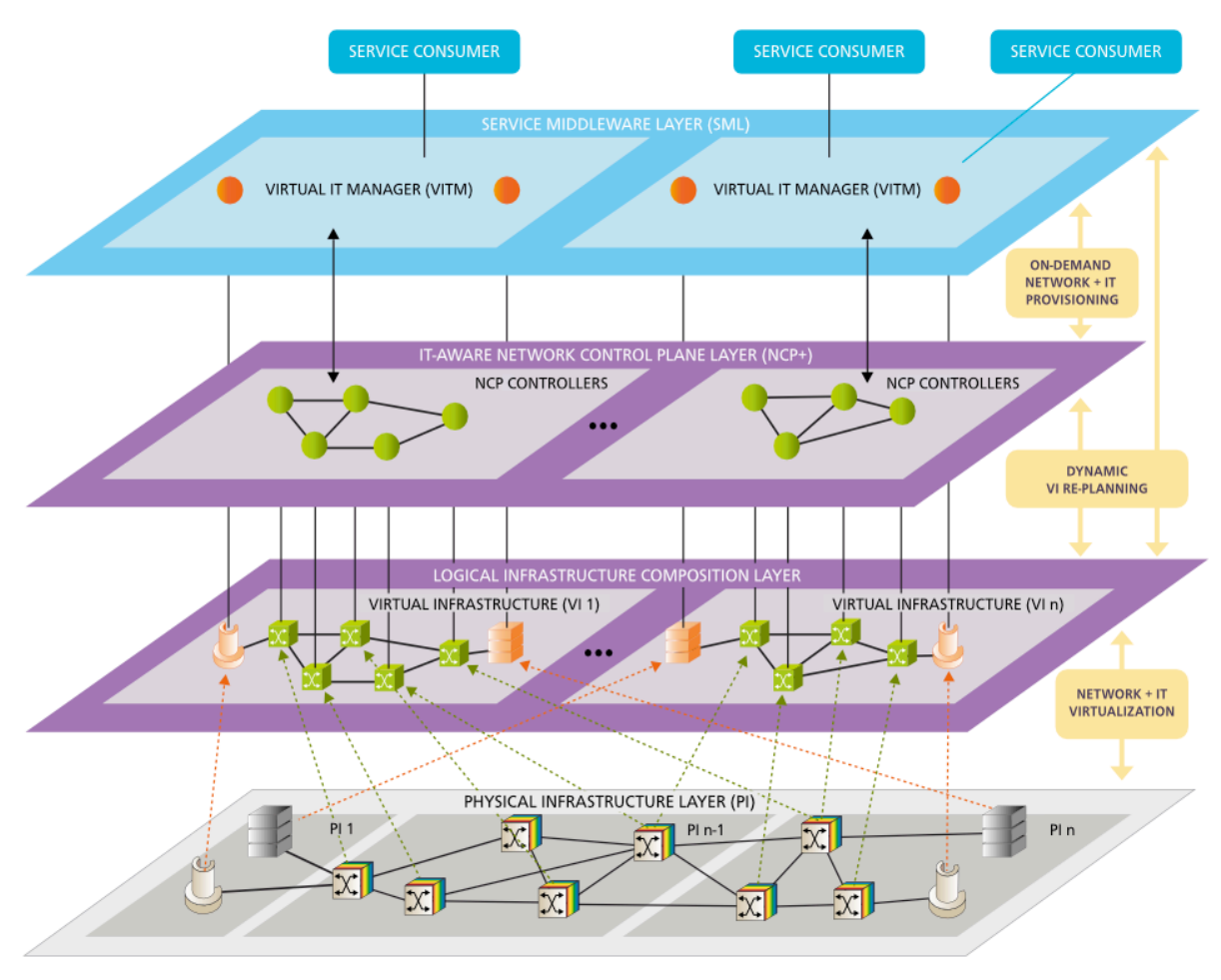

Figure 1: GEYSERS architecture and related value chain roles.

The LICL plays a crucial role in the GEYSERS architecture, as it is responsible for the planning and allocation of virtual infrastructures (VI) composed of virtualized network and IT resources (VR) [12]. On the one hand, the LICL is responsible for resource abstraction, resource publishing, VR creation and management, and VR operation. On the other hand, it also deals with VI creation, management and replanning. Figure 1 depicts the internal representation of the LICL, with several virtual infrastructures composed of different virtual resources - i.e, the green and orange boxes - representing virtualised partitions of the physical resources at the bottom level.

The NCP+ operates over virtual infrastructures composed by the LICL allowing virtual operators to offer on-demand and coupled provisioning of the IT resources and the transport network connectivity associated to IT services. The NCP+ designed in GEYSERS is an enhancement of the ASON (Automatic Switched Optical Network) / GMPLS (Generalized Multi-Protocol Label Switching) control plane, as it relies on newly defined extensions to the RSVP-TE signalling protocol and the OSPF-TE routing protocol. Figure 1 contains the representation of the different $\mathrm{NCP}+$ controllers - green circles - deployed over a given set of virtual infrastructures at the $\mathrm{NCP}+$ level. The $\mathrm{NCP}+$ also includes a Path Computation Element (PCE + ) for enhanced routing decisions on the virtualized optical network: the network service routing in GEYSERS NCP+ is obtained via an enhanced PCEP protocol and routing algorithms mix of Traffic Engineering policies for optical networks with energy-efficient heuristics [13]. The combined Network and IT Provisioning Service (NIPS) is performed through a service-to-network interface, called NIPS UNI. Over the NIPS UNI, the NCP+ offers functionalities for setup, modification and tear-down of enhanced transport network services (optionally combined with advance reservations), monitoring and cross-layer recovery and for the advertisement of IT resources from the SML. Details on the exact design of the NCP+ functions, services and GMPLS/PCE protocol extensions can be found in [14][15][16]. 
Finally, the SML is a convergence layer for requesting and partially managing a customized virtual infrastructure for a specific application or market segment characterized by specific application needs such as processing, storage, memory, power and connectivity. The orange circles represented in the Figure 1 at the SML level comprise the different Virtual IT Managers of the SML devoted to manage the lifecycle of the virtual IT resources that are used by the different applications served by the SML. An application request at the SML includes constraints that either explicitly or implicitly state these needs. These constraints are the set of Service Level Objectives (SLOs) when the request is issued. They are reflected in the Service Level Agreements (SLAs) between providers and consumers once the resources are selected and provisioned through the NIPS service to handle the request [17]. The SLAs contain the performance invariants in terms of response times and load distribution. The SLAs are also used for scaling the computational and network resources of the virtual infrastructure provisioned through the LICL layer. In GEYSERS we defined the process of creating a service request and handling that request within a resource management infrastructure. A request therefore implicitly embodies an SLA. The changing status of handling such a request represents the handling and status of the SLA. Further details on the specifics of SLA management through the GEYSERS architecture can be found in [23].

\subsection{GEYSERS business roles}

The GEYSERS layers enable the adoption of different roles according to the specific functionalities supported by the architecture, namely the Physical Infrastructure Provider (PIP), the Virtual Infrastructure Provider (VIP) and the Virtual Infrastructure Operator (VIO).

The PIP is the owner of the physical resources, i.e. both network (optical nodes and links) and IT resources (CPUs, storage and memory), typically associated with the carriers and telecom operators. By leasing virtual resources, a PIP is able to efficiently utilize its infrastructure and obtain an increased benefit from its rollout, thus achieving a faster return on investment.

The VIP is the infrastructure broker. It offers virtual infrastructures composed of virtual network and IT resources, possibly from several PIPs, according to the requirements of its clients. Being able to provide customized, on-demand virtual infrastructures, the VIP offers more attractive services and increases its competitiveness.

The VIO rents virtual infrastructures and offers unified services over dedicated infrastructures to the end users. This way, the VIO is able to reduce capital expenditure, operational costs and time-to-market. Moreover, the VIO can dynamically adjust the virtual infrastructure according to his needs, thus benefiting from a pay-as-you-grow model.

\section{GEYSERS Future Internet Testbed}

In order to validate the GEYSERS architecture and prototypes, the project deployed a physical testbed infrastructure composed of administratively independent local testbeds. These local testbeds, provided by several project partners, are interconnected through GÉANT and National Research Education Networks (NRENs). In total, testbeds from seven countries (and ten partners) have been interconnected, including infrastructure across Belgium, France, Italy, Poland, Spain, the Netherlands and the United Kingdom (Figure 2). 


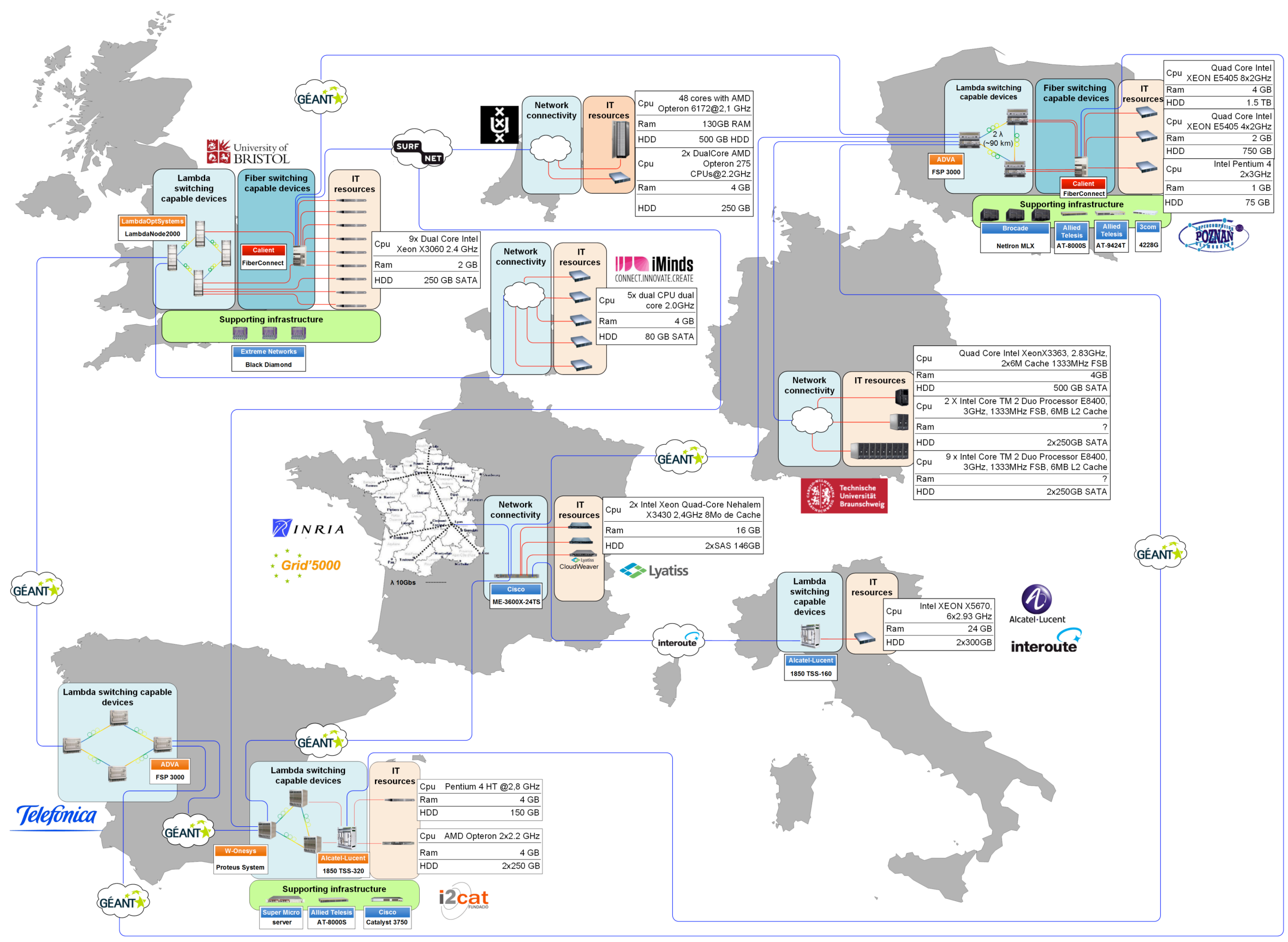

Figure 2: Overview of the GEYSERS testbed 
This testbed contributed by GEYSERS partners served as a validation tool for the GYESERS architecture and was not conceived, at least at the time of creation, to run as a FI experimental infrastructure like the ones developed through other initiatives, e.g. FEDERICA Error! Reference source not found., OFELIA [30] or GENI Error! Reference source not found..

Local testbeds are mainly composed of optical equipment and IT resources (CPU and storage). Partners also provide supporting Layer-2 infrastructure to enable: (i) the communication between the IT resources and the optical infrastructure, and (ii) the inter-testbed VLAN-based communication across GÉANT and the NRENs. However, different partners contribute with different types of physical resources to represent the heterogeneous nature of a global physical infrastructure.

The mix of optical and Ethernet switching technologies deployed in the GEYSERS testbed has been assembled to replicate a real word network deployment scenario for the proof and assessment of the main concepts/functions designed in LICL and NCP+. In particular, the GEYSERS testbed presented in this section can uniquely demonstrate the key project innovations in two main areas:

- At the infrastructure and network management level: partitioning of the physical infrastructure and its logical composition into multiple logical infrastructures, each one operated with a Network Control Plane by a network operator. LICL is the main driver in this area.

- At the transport connection provisioning level: enhancements to the Network Control Plane (starting from the standard ASON/GMPLS and PCE architectures) for coupled Network+IT transport services. GMPLS + PCE + are the main drivers in this area.

The testbed built as described below represents a key asset for such aforementioned experiments, and its impact is further strengthened by the use of networking hardware in real use within Network Operators and NRENs infrastructures.

\subsection{Guidelines for building the GEYSERS geographically distributed testbed}

The creation of a GEYSERS testbed required the following steps:

- Definition of the distributed local testbeds composing the overall physical infrastructure, including the complete specification of their network and IT capabilities.

- Deployment and configuration of the internal optical networks within each local testbed. In GEYSERS scenarios, both fibre-switching and lambda-switching technologies are supported. The decoupling of the control plane from the physical devices, enabled through the virtualization layer, allows to easily integrate a wide range of equipment, independently of their specific management protocols and interfaces. The LICL software already includes adaptors for Calient DiamondWave Fiber Connect optical switches, ADVA FSP-3000 equipment and Alcatel-Lucent 1850 packet-optical transport switches. Dedicated LICL adaptors need to be developed in order to support additional types of equipment.

- Deployment and configuration of the inter-connectivity between the local testbeds for the data plane and the Signalling Communication Network ( $S C N)$ related to the control plane. Further details about these types of inter-connectivity mechanisms are provided in Section 3.3. 
- Deployment and interconnection of computing and storage resources within each local testbed (see Section 3.4 for further details). IT resources are connected to the optical networks through a supporting network infrastructure based on L2 devices.

- Installation and configuration of LICL and NCP+ components on the testbed. LICL and NCP+ prototypes are delivered as two VM images, each of them collecting all the different components of LICL and NCP+ respectively (which can be downloaded from http://sourceforge.net/projects/geysers-ncp/ and http://sourceforge.net/projects/geysers-licl/). A dedicated VM must be deployed for each LICL or NCP+ component planned in the environment; all the VMs must run on servers connected to the SCN. Further details about LICL and NCP+ deployment and configuration are available in the respective sourceforge project repositories. In a typical scenario with a single Virtual Infrastructure composed of resources from multiple PIPs and operated as a single routing domain at the $\mathrm{NCP}+$ level, the following software components must be deployed:

- A LICL instance for each PIP offering physical IT or network resources.

- A LICL instance for each VIP.

- A GMPLS controller for each optical node in the Virtual Infrastructure.

- A child PCE server.

- A NIPS server.

- An AAA server.

- An SML instance.

In case of multiple routing domains, each routing domain connecting IT resources must have its own NIPS server. Moreover, a child PCE server must be deployed for each (edge or core) routing domain, together with an additional, centralized parent PCE server.

\subsection{GEYSERS local testbeds}

This section presents details of the local testbeds contributed by GEYSERS partners to the project. As already stated, both Lambda and Fibre switching capabilities (LSC/FSC) are available within the GEYSERS testbed, together with a number of servers well distributed among the partners. Table 1 summarizes the testbed resources provided by each partner that are controlled by the GEYSERS control plane. Furthermore, additional servers are required in all local testbeds to deploy the prototypes of the GEYSERS stack: LICL, NCP+ components and SML.

Table 1: Physical resources contributed by each project partner

\begin{tabular}{lll}
\hline Partner (Country) & Network resources (Vendor) & IT resources (Vendor) \\
\hline i2CAT (Spain) & 3x Optical LSC Nodes (W-Onesys) & 2x Servers (SunFire \& Dell) \\
iMinds (Belgium) & - & $5 x$ Super Micro + 1x NFS \\
IRT/ALU (Italy) & 1x Packet-Optical Transport Switch (ALU) & 1x Workstation (HP) \\
LYaTiss (France) & - & 2x Servers (Dell) \\
PSNC (Poland) & 1x Optical FSC Node (Calient) & - \\
TID (Spain) & 7x Optical LSC Nodes (ADVA) & 2x Servers (Dell \& HP)
\end{tabular}




$\begin{array}{lll}\text { UvA (Netherlands) } & - & 1 x \text { Server (Dell) } \\ \begin{array}{l}\text { University of Bristol } \\ \text { (UK) }\end{array} & \text { 1x Optical FSC Node (Calient) } & \text { 2x Servers (Dell) }\end{array}$

To highlight the work done within the local testbeds and as an example of specific devices, ports and internal connections that may be present at each of these local testbeds, two local testbeds are further depicted below. The complete specification for each testbed can be found in [19].

\subsubsection{University of Bristol local testbed}

The devices deployed at University of Bristol premises are depicted in Figure 3. They consist of a Calient DiamondWave Fiber Connect optical switch that provides the FSC data plane infrastructure. The Calient is manually pre-partitioned into 4 sub-switches to form a simple mesh network topology. University of Bristol also provides 8 DELL PowerEdge 860 servers, 6 of which are used for the deployment of the GEYSERS software (LICL and NCP+ controllers) and the other 2 compose the PIP-IT part of the testbed. Three supporting devices (Extreme Blackdiamond 12804, DELL Powerconnect 2716 and a FOUNDRY FastIron Edge X424HF) provide data plane connectivity to remote testbeds through GÉANT, and control plane connectivity to the GEYSERS Signalling Communication Network (SCN).

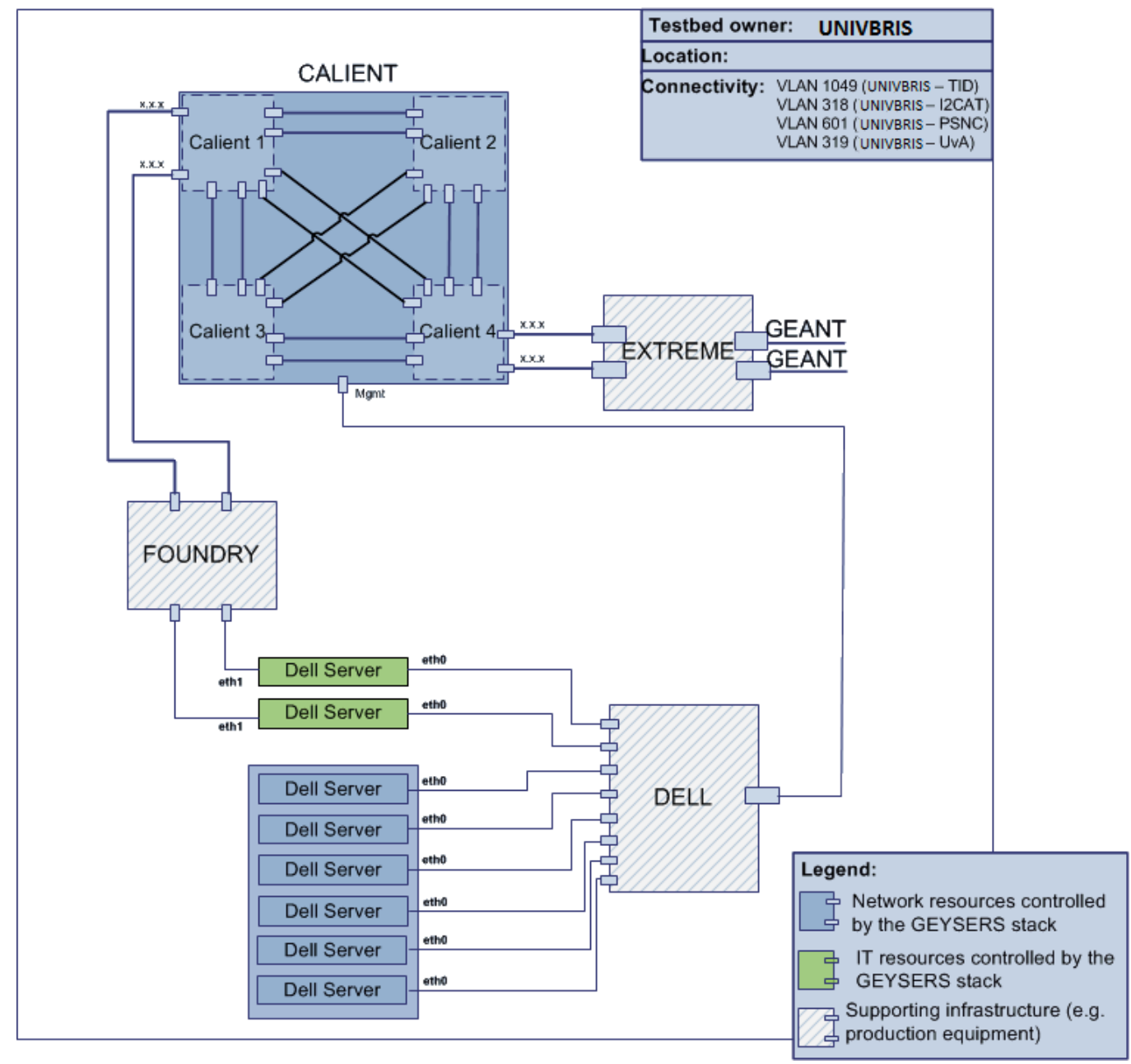

Figure 3: University of Bristol local testbed facilities 


\subsubsection{Fundació i2CAT local testbed}

The testbed provided by i2CAT is shown in Figure 4. Two SuperMicro servers are used for deploying the LICL prototype and supporting software (e.g. OpenNebula). A SunFire x2200 server, a Dell PowerEdge 850 , and 2 additional SuperMicro servers act as IT resources. The configuration of these IT resources is carried out through a Lyatiss CloudWeaver machine.

The network resources consist of three W-Onesys Proteus devices. Two Cisco Catalyst switches and an Allied Telesis switch compose the supporting infrastructure to provide the data plane connectivity within this testbed, towards other GEYSERS testbeds and for the SCN connectivity.

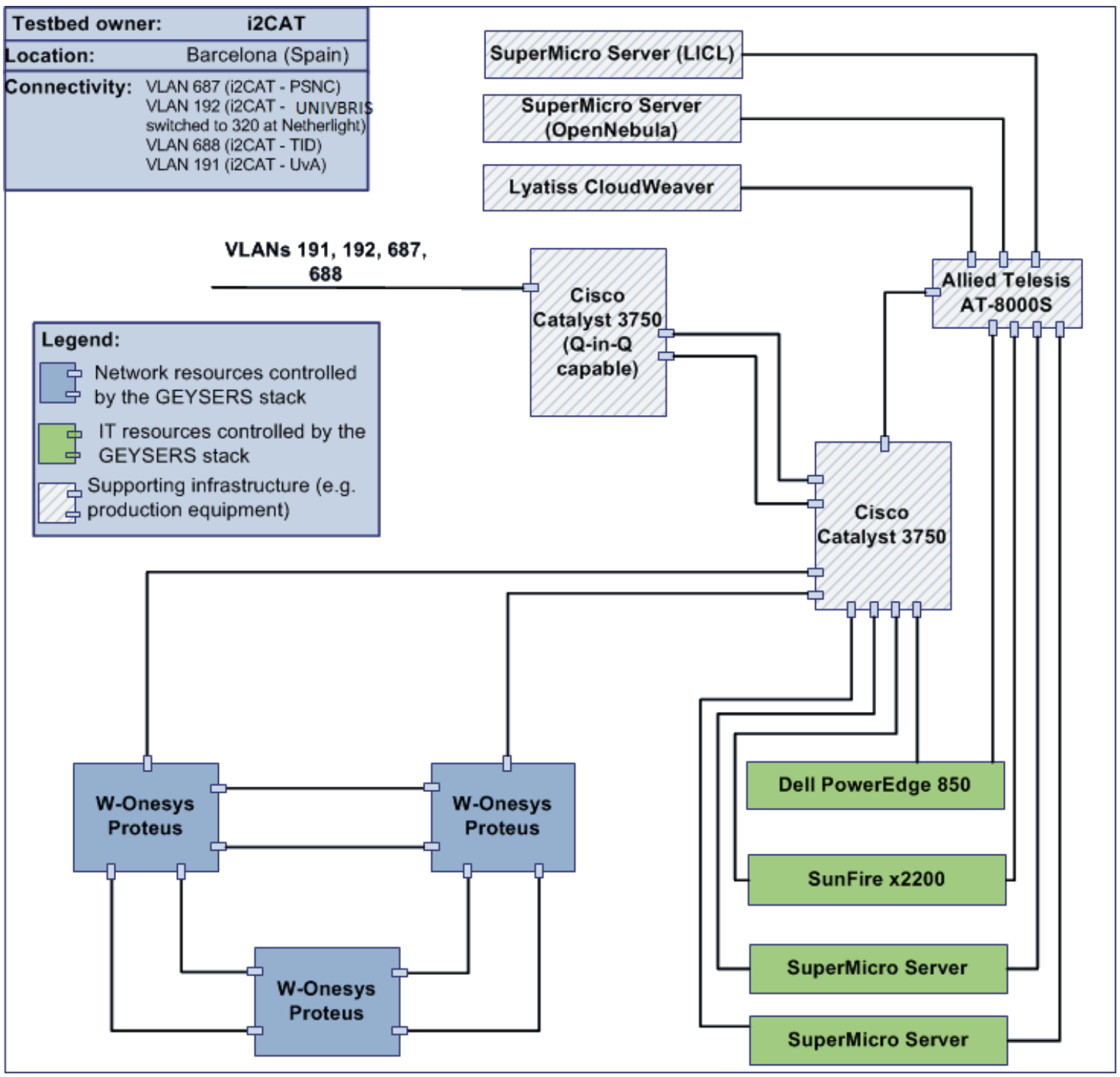

Figure 4: Fundació i2CAT local testbed facilities 


\subsection{Interconnectivity between local testbeds}

GEYSERS local testbeds are not isolated, but interconnected among them, as shown above in Figure 2. The GEYSERS testbed has been designed and deployed taking into account a control and data plane separation.

Signalling among the GEYSERS local testbeds comprises communication between deployed instances of the LICL, SML and the NCP+ network controllers. These instances are deployed at computing facilities located at different, geographically distant locations. To handle an exchange of signalling/routing messages the control/management layer has to be isolated from the external traffic and safe from communication interruptions. To meet these requirements a dedicated Signalling Communication Network $(\mathrm{SCN})$ is introduced in the GEYSERS testbed.

A separation of the SCN and data plane brings a number of benefits to potential users of the GEYSERS testbed. First of all, the SCN allows each partner to assign private IP addresses to the hosts on which control and management entities are deployed. The use of private IP address pools for the SCN increases the network security, as the control stack is not directly vulnerable to attacks from the Internet. Second, the separation allows the control stack to manage physical devices located in local testbeds without the need for the reorganization of each local network addressing scheme. Finally, the usage of a dedicated network for signalling provides the full isolation of data and control plane links and flows. Any potential failure of a data plane link does not affect the state of the GEYSERS control stack. Moreover, the control and signalling mechanisms may be tested and validated regardless of the status of the data plane connectivity, e.g. inter-domain data links.

\subsubsection{Data plane interconnection}

The GEYSERS physical infrastructure includes not only the switching resources and IT facilities deployed at the local testbeds, but also the international optical links interconnecting them. In most cases, the lightpaths between the GEYSERS local testbeds are provided by the GÉANT infrastructure. In those scenarios, services from local NRENs (e.g. SURFnet, RENATER, RedIRIS, Belnet, JANET) are used in order to reach the closest GÉANT Point of Presence (PoP). The few exceptions to this common situation include the link between LYATISS and IRT, which is deployed over Interoute's optical network; PSNC, which is directly connected to GÉANT also shares a common access link with TP, and the link between University of Bristol and iMinds is shared with the OFELIA project [20].

The interfaces at local testbeds are 1 Gbps Ethernet interfaces. The nodes connected through GÉANT rely on the GÉANT Plus service [21], which enables point-to-point circuits with guaranteed bandwidth and a deterministic performance over a pre-provisioned network. In other words, the GÉANT Plus service can be seen as a private line over a common infrastructure.

In order to increase the testbed flexibility and accommodate different test scenarios in a cost effective way, multiple Ethernet VLANs on a single physical link are used. The approach brings the following benefits to the project: (i) establishment of logical connections between local testbeds which are not directly connected is possible, and (ii) multiple virtual links between testbeds may be used to perform experiments in parallel. 
However, it requires the physical links to be terminated on Gigabit Ethernet switches, where the VLANs separation happens. An IEEE 802.1 ad (i.e. stacked VLANs, also known as Q-in-Q) solution has been selected as a preferable solution when supported. By using Q-in-Q, multiple users' VLANs can be encapsulated into a single one to be transmitted over the lightpath. Consequently, the testbed provider is not involved in the distribution of bandwidth among the VLANs used by experiments, and any reconfiguration needed for an experiment is easier to manage.

Figure 5 presents the interconnection links between local testbeds in the GEYSERS project.

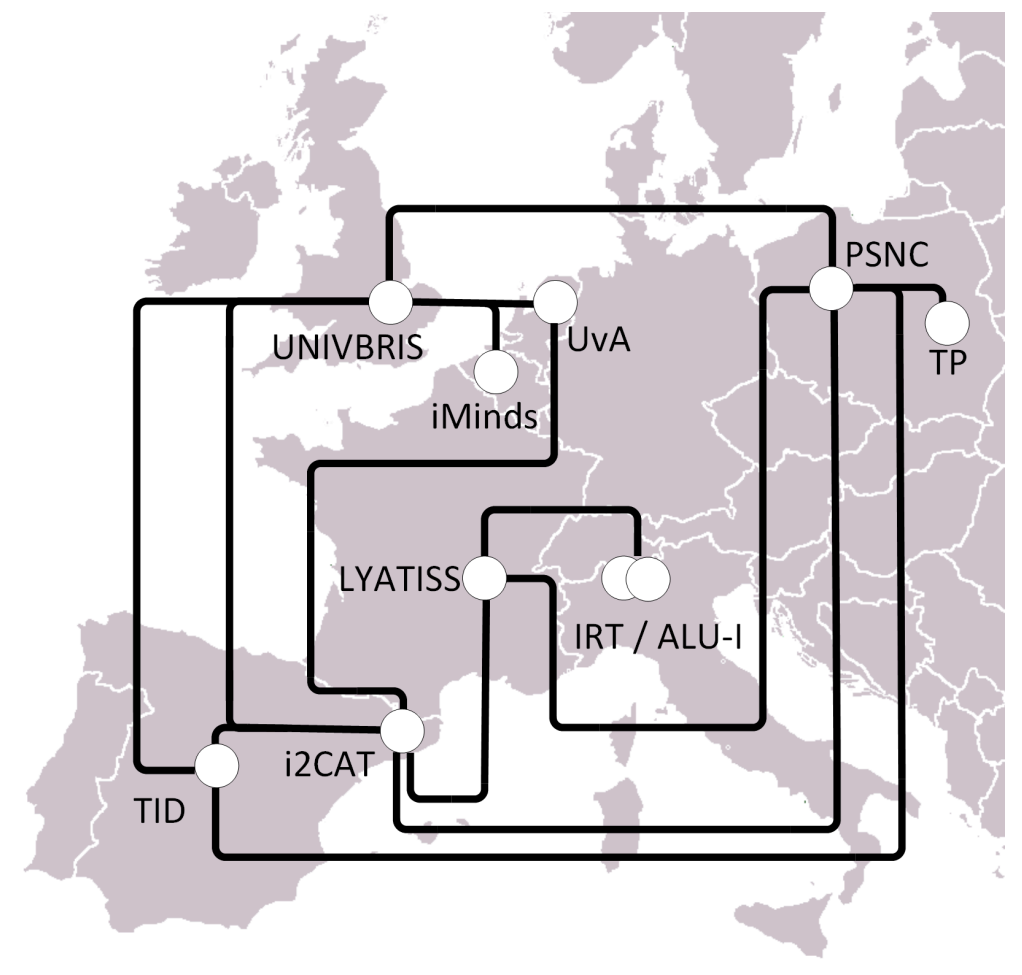

Figure 5: Data plane interconnectivity between local testbeds in GEYSERS

\subsubsection{GEYSERS Signalling Communication Network (SCN)}

Communication among the deployed instances of the GEYSERS software stack, which are installed on servers located at different sites, is enabled at the control and management planes by means of the project's Signalling Communication Network (SCN).

Figure 6 shows its current topology: a star. The SCN Main Router is hosted at PSNC premises, while SCN Access Routers are deployed at each partner's local testbed (in PSNC as well). Routers located in local facilities are interconnected over the Internet or through GÉANT to the central router. GRE (Generic Routing Encapsulation) technology is used to provide IP tunnels over the Internet. Consequently, each partner can use private address pools for control and management purposes, avoiding unnecessary assignment of new public addresses, and is not vulnerable to attacks from the Internet.

Each SCN Access Router is deployed as a virtual machine and acts as a router for local control and management instances to enable inter-domain communication. To facilitate the deployment, a virtual 
machine image has been prepared with proper configuration files that are activated during the boot of the virtual machine. The configuration file is used to set a proper network configuration of the host being the gateway between the local and remote testbeds, and is based on static entries to the routing table of each host participating to the SCN network.

Additionally, in order to manage the GEYSERS stack remotely (for instance, during demonstrations) an OpenVPN server was installed at the SCN Main Router. Therefore, on each host that needs to communicate through the SCN, an OpenVPN client needs to be configured as well, with the proper credentials.

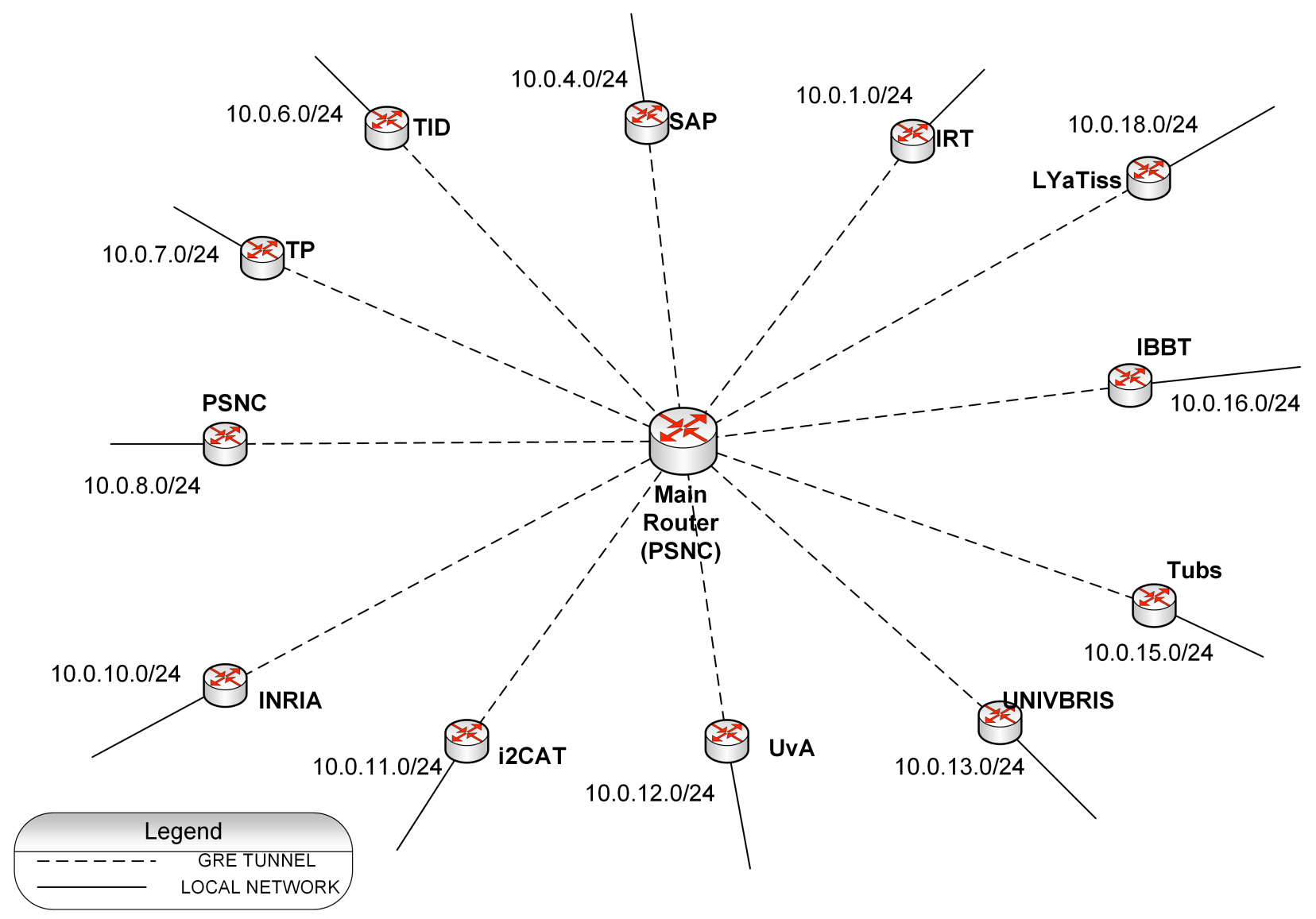

Figure 6: The Control/Management Plane topology in the GEYSERS test- bed

\subsection{Interconnectivity of IT resources to the network}

Typically, IT resources are located in data centres or warehouses connected to the backbone network infrastructure through a separate L2 or L3 supporting network. In the GEYSERS testbed, the virtualization of this separate network is out of scope of development and it is effectively carried out by the LICL using supporting software such as OpenNebula [22] and/or CloudWeaver [23] functionalities, with interfaces that also support the actual IT resource provisioning. In order for the virtual IT resources (Virtual Machines (VMs)) controlled by OpenNebula to be connected to the actual network infrastructures several requirements need to be met, starting from physical related ones (like connecting the servers to VLANtrunk enabled switches) and continuing with software requirements for the configuration of the servers 
which will be used for hosting the VMs. Figure 7 summarises how the VMs are actually connected to the physical network.

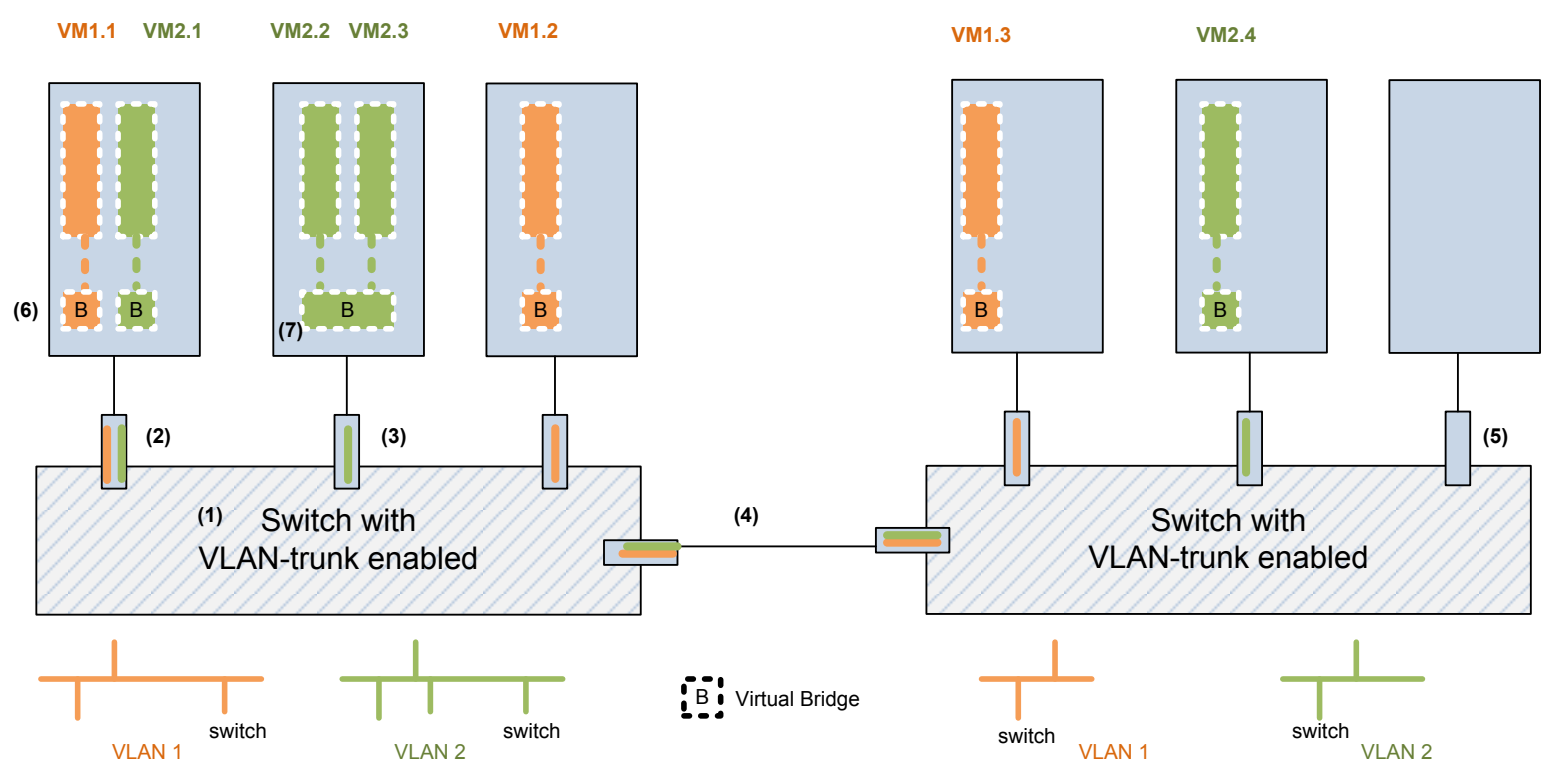

Figure 7: VLAN enabled infrastructure across multiple switches

(1) Each switch used to connect VMs in a virtual infrastructure must be VLAN-trunk enabled.

(2) Ports on a switch are tagged with VLAN Identifiers such that broadcast messages intended for specific VLANs are only sent to those ports.

(3) Ports may be tagged for a single VLAN, enabling high isolation of network traffic and management broadcast messages.

(4) Ports used for connecting switches must also be tagged with the respective VLAN Identifiers.

(5) Only ports that are active in virtual infrastructures need to be enabled, unless used for non-VLAN traffic.

(6) VMs are attached to virtual bridges via their virtual network interface (vNIC). Note that a VM may have multiple vNICs each connected to different virtual bridges, if the VM is to be connected to multiple VLANs.

(7) VMs on the same physical host and VLAN share the same virtual bridge

Each VM uses at least one virtual network interface for connecting to a virtual network bridge. In case the virtual network bridge does not exist, OpenNebula will create one and attach it to a physical network interface tagged with a VLAN-ID. The OpenNebula Adapter ensures that for each logical resource a different VLAN-ID will be used, in this way assuring that the VMs belonging to the same virtual infrastructure will be able to communicate between them, but not with the VMs belonging to different virtual infrastructures.

As the IEEE 802.1q [24] mechanism is being used, certain configurations need to be performed on the servers hosting the VMs, such as installing the kernel module 802.1q and the vconfig package, and also allowing the user under which OpenNebula connects to execute vconfig, brctl and IP commands in the host. The physical switch to which the host is being connected must support VLAN-trunk protocol. 
Given the fact that one of the physical network interfaces in the hosts will be used for carrying VLANtagged traffic, it is recommended that at least two different network interfaces exist in hosts. The second one would permit remote management operations (e.g. configuration and monitoring), for example allowing the VM to connect to Internet (if desirable).

\section{Experiments in the GEYSERS testbed}

The GEYSERS testbed hosts different kinds of experiments: (i) prototype integration, (ii) architecture validation, and (iii) demonstration. First, the integration of prototypes comprises validation and collaboration between particular prototypes as system components. This integration assures that specific overall system components are able to collaborate smoothly, and perform their tasks according to the expectations defined during the design and implementation processes. Second, an overall architecture and deployment validation of the system as a whole covers collaboration between all GEYSERS prototypes, network environments, and application layer. Finally, the testbed demonstrates prototypes features and operability to communities addressed by the GEYSERS project.

The integration of the GEYSERS architecture components on the testbed aims at showing its ability to jointly provision network and IT services on a real multi-site infrastructure, and its integration with current cloud-based infrastructure service provisioning technologies. The value of the GEYSERS approach is shown on the testbed using four demonstrators highlighting the benefits of GEYSERS architecture in real use cases. The experiments evaluate the model both for all the key technical dimensions and from the usage/business perspectives of the involved actors, focusing on different components and/or functionalities offered by the GEYSERS architecture.

- The focus of Demonstrator 1 is to demonstrate the LICL functionalities through the network virtualization service offered by an Infrastructure Provider to a Virtual Infrastructure Operator (VIO), and how the involved players interact. This scenario aims at achieving a better usage of the available resources through its virtualization, thus reducing the capital expenditure (CapEx) and operational costs (OpEx).

- Demonstrator 2 shows the on-demand provisioning of enhanced network connectivity services, tailored to the specific requirements of the applications, over a Virtual Infrastructure. The technical focus of this demonstrator is on the $\mathrm{NCP}+$ functionalities that support the different types of specialized connectivity services that a VIO-N is able to provide to its customer (typically a VIO-IT).

- The objective of Demonstrator 3 is to dynamically adjust the available computing, storage and network resources for a cloud based Enterprise Information System (EIS) based on the demand from the users of the EIS. This demo illustrates methods of dynamic synchronized scaling of both IT infrastructure and network capacity. We assess the benefits for cost and overall "satisfaction" of the EIS provider's operational objectives, without disrupting the application user's service level objectives and experience.

- Demonstrator 4 focuses on the advanced network and IT management functionalities, with particular attention to network infrastructure re-planning. The virtual infrastructure re-planning service allows the VIO to request the modification, up- or down-scaling (e.g. upgrade of link capabilities, modification of network topology) of the leased virtual infrastructure in order to optimize the network resource utilization. 


\subsection{Demonstrator 1: Virtual Infrastructures for Virtual Infrastructure Operators}

Demonstrator 1 shows the automatic provisioning process of virtual infrastructures in multi-domain scenarios. This demonstrator is based on two premises: (i) infrastructure owners would use virtualization to provide access to their physical infrastructures to third parties, thus making a better use of the available resources, and (ii) a VIO prefers to rent a part of a physical infrastructure (which in turn instantiates a virtual network) instead of deploying a network infrastructure on its own, thus reducing the capital expenditure and operational costs.

The main goal of Demonstrator 1 is to present the capabilities of the GEYSERS LICL. In a nutshell, the LICL supports virtualization of both, network and IT resources, and automatically provides virtual infrastructures by dynamically selecting and composing virtual resources pertaining to different infrastructure providers. The LICL offers the fundamental basis for the other layers from the GEYSERS architecture (i.e. NCP+ and SML) to provide enhanced services, adapted to the user's requirements. PIP, VIP and VIO roles are involved in the context of this demonstrator.

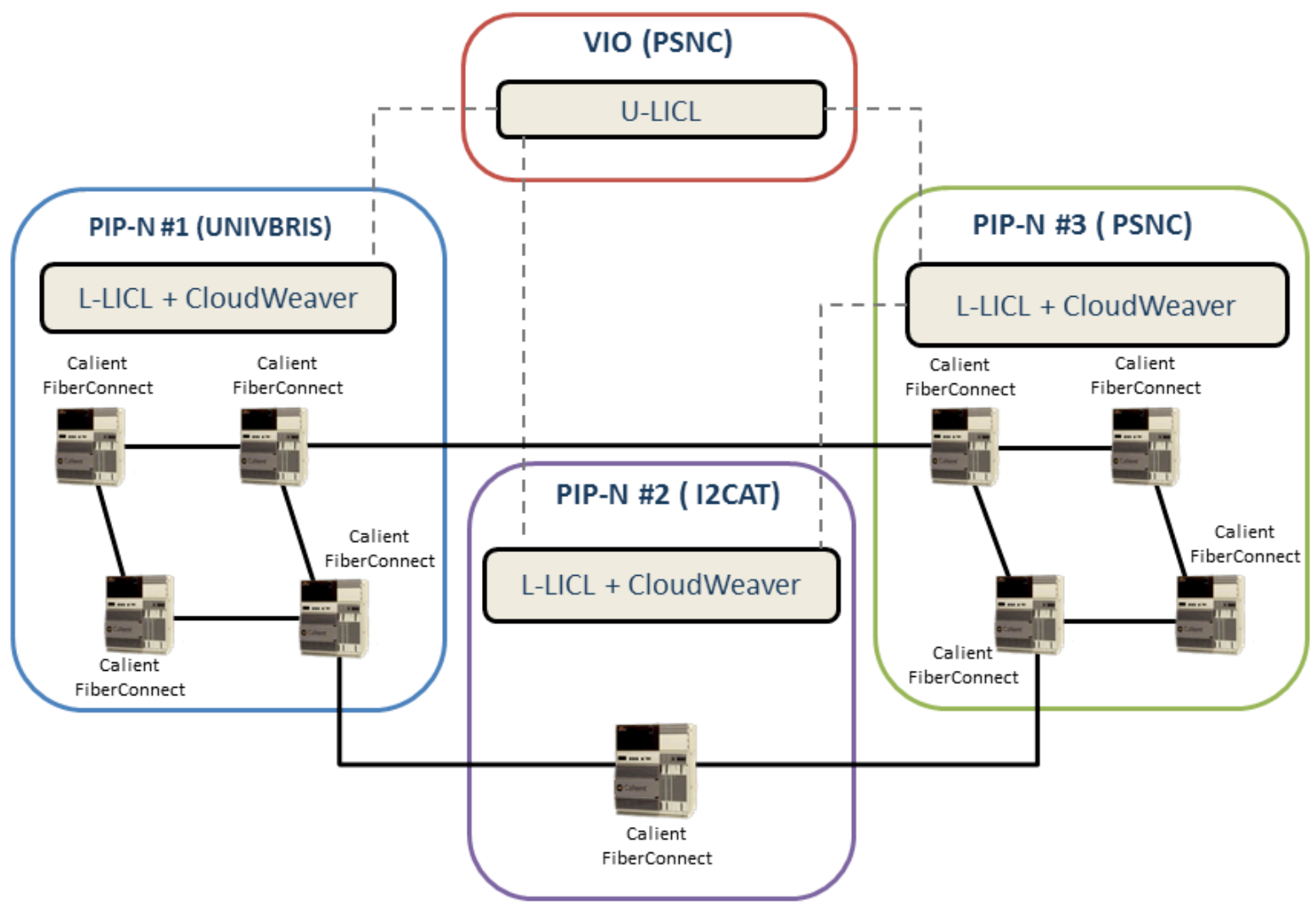

Figure 8: Resources and roles in Demonstrator 1.

Figure 8 depicts the physical infrastructure assigned for the demonstration and the involved GEYSERS roles. In this scenario, University of Bristol, i2CAT and PSNC play the role of PIPs and provide network resources for virtualization by deploying the lower-LICL and the CloudWeaver software. PSNC also assumes the role of VIP, and consequently also deploys the upper-LICL software. 
This demonstrator has been implemented and successfully shown at FuNeMS held in Berlin in July 2012 [25]. During the demonstration, basic functionalities of the LICL have been shown, such as PIP registering physical resources with the lower-LICL, on-demand creation of a virtual infrastructure and its subsequent instantiation. Over the instantiated virtual infrastructure, cross-connections have been created and several ping operations have been executed between hosts attached to the infrastructure. Finally, the virtual infrastructure has been decommissioned and the resources have been released to the system, thus making them available for future use. Additionally, during the demonstration, the user interface provided by the CloudWeaver has been used to show the status of the resources allocated to the created virtual infrastructure.

\subsection{Demonstrator 2: Anycast Networks in Virtual Infrastructures}

The second GEYSERS demonstrator is focused on the convergence and coordination between network and IT resource provisioning. In particular, this demonstrator shows the functionalities of the GEYSERS Network Control Plane $(\mathrm{NCP}+)$ to operate the virtual infrastructure through the interface exposed by the LICL and to provide enhanced network services in cooperation with the SML.

Beyond the unicast services traditionally offered by current network operators, this demonstrator shows new service paradigms where the network connectivity is dynamically adapted to the application requirements optimizing the combined allocation of the mixed set of resources (i.e. network and IT) composing the virtual infrastructure. This feature is enabled through the cross-layer cooperation between SML and $\mathrm{NCP}+$, where the selection of the IT end-point is performed taking into account network conditions and cost, and is progressively delegated to the NCP+ in the anycast case. This concept allows the GEYSERS architecture to offer an integrated control of the end-to-end service along the different phases of its lifecycle, from its establishment to its deletion, supporting mechanisms for cross-layer recovery in case of accidental resource failures. This approach offers several benefits for both customers and providers. On one hand, the (virtual) network infrastructure operators will be able to optimize the utilization of their (leased) network by providing services specifically tailored to the dynamic requirements of their customers. On the other hand, customers such as cloud service providers will receive network services customized for the required QoS and resiliency guarantees at competitive cost, with opportunities to apply SLAs that can be dynamically updated following the changing requirements of their own businesses.

Figure 9 shows a Demonstrator 2 overview: the physical infrastructure is composed of network resources from the University of Bristol and PSNC testbeds and IT resources from the University of Bristol, TID and TP testbeds. In each one of these testbeds, representing the infrastructures owned by PIPs, the lowerLICL has been deployed to allow the virtualization of the resources. The TID testbed includes as well the upper-LICL components that provide the composition of the virtual infrastructure. This virtual infrastructure is then rented from the VIP, represented by TID, to the VIO. This role is represented by the University of Bristol, whose testbed has been consequently used to deploy the GEYSERS components related to Network Control Plane $(\mathrm{NCP}+)$, and is responsible for operating and providing the enhanced network services described above over the virtual infrastructure. 


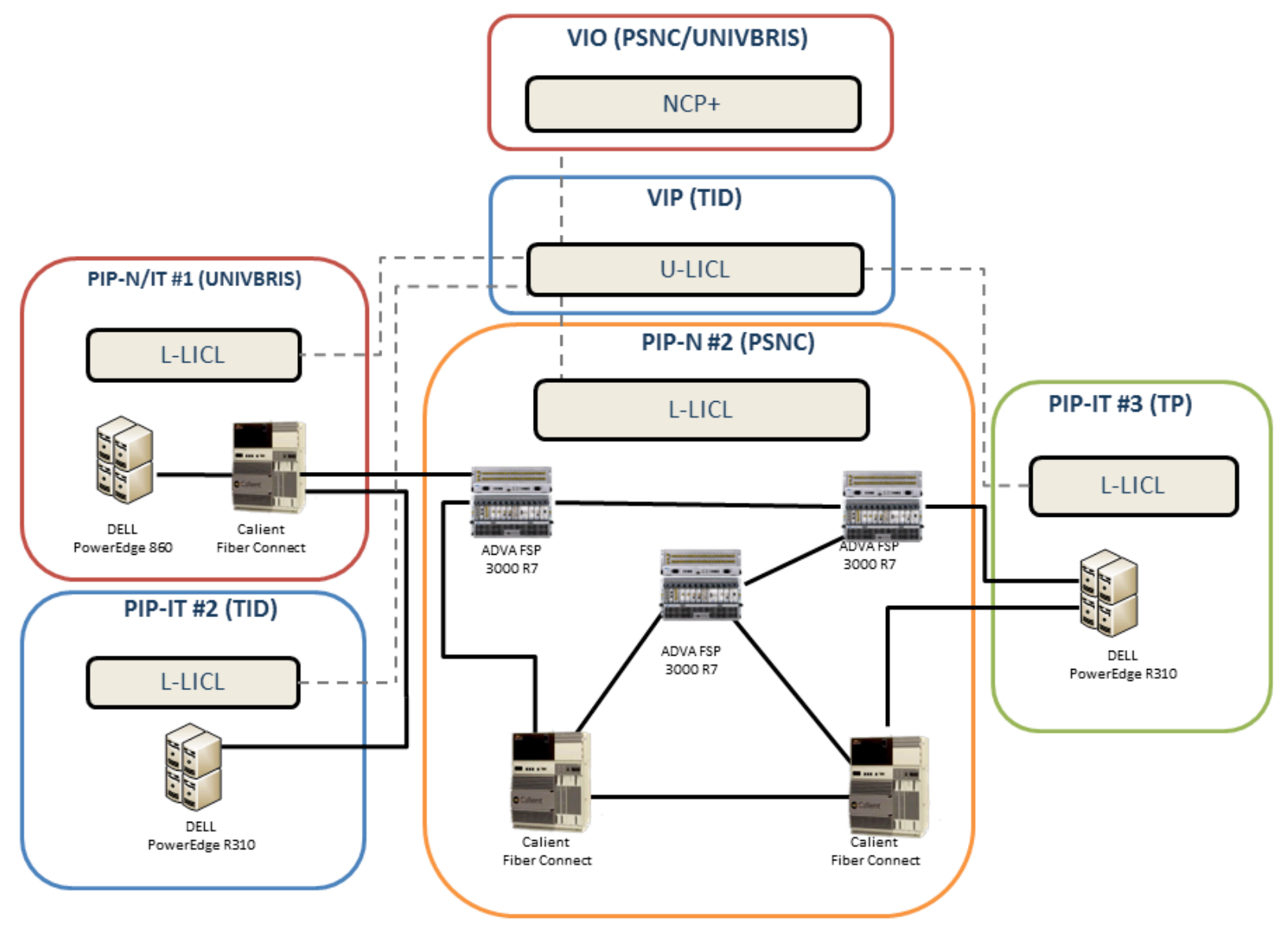

Figure 9: Resources and roles in Demonstrator 2.

As well as Demonstrator 1, an initial version of this demonstrator has been presented during the FuNeMS'12. In this event, we demonstrated the NCP+ procedures for the on-demand setup and tear-down of different types of connectivity services over a single-domain virtual infrastructure. In the unicast service, the traditional GMPLS and Path Calculation Element (PCE) [26] based functionalities of the $\mathrm{NCP}+$ have been shown. This initial service, where network and IT resources are considered as separate entities and not jointly processed, contrasted with the most efficient scenario of the anycast services also demonstrated. In this case, the selection of the IT end-points is fully delegated to the NCP+ that is able to optimize the overall resource utilization, searching for storages/servers compliant with the description specified in the application requests and, at the same time, reachable through efficient network paths providing the required bandwidth. In this context, we showed the innovative procedures in support of IT advertisement mechanisms to make the $\mathrm{NCP}+$ aware of the capabilities/availabilities in the resources located at the IT end-points, the RSVP-TE signalling extended for the control of the NIPS call and the NIPS path computation at the centralized PCE, with the anycast routing algorithms to jointly select the network and IT resources [16]. 


\subsection{Demonstrator 3: Cloud-based Enterprise Information Systems}

Demonstrator 3 shows the possibility and value of dynamically adjusting the computing, storage and network resources of a virtual infrastructure based on the needs of an Enterprise Information System (EIS) consuming it. Methods and mechanisms for dynamically synchronizing the scaling of IT infrastructure and network capacity are demonstrated. An EIS is a query-intensive application-server and database system that serves various concurrent users. In a dynamic EIS the number of users and frequency of queries changes, such that the network and resource demands change as well. The capacity of the network and servers involved should then reflect this demand without being over-provisioned. The number of EIS users is increased and decreased over time, as well as the frequency and size of the request payloads and transaction workloads executed concurrently. The implementation of EIS used is an emulator of workload and payload classes used to control this variation of concurrent users and transactions. An existing Online Transaction Processing (OLTP) benchmark is used as the basis for the workload representation.

The starting point for the demonstrator is the SML, which provides functionalities for accessing a virtual infrastructure and defining application-centric control rules. The demonstrator hence uses the entire GEYSERS stack in a top-down manner to achieve a decoupling of virtual infrastructure policy from the configuration of virtual and physical resources that compose it. The LICL is used to coordinate the actions resulting from the SML's rules, and to request the necessary changes to the underlying resources. The benefits for cost and overall "satisfaction" of the EIS provider's operational objectives, without disrupting the application users' service level objectives and experience are assessed.

Figure 10 shows the overall Demonstrator 3 picture. This demonstrator involves the VIO, VIP and PIP roles in the GEYSERS framework, but features the Application Provider (APP) role to initiate the interactions. SAP facilities trigger the APP requests access to a virtual infrastructure for hosting the EIS as a service, such that there is no need for the APP to own computational resources and high-speed network equipment on their premises, following the cloud computing model. The goal is for the APP to only focus on managing the Service Level Objectives (SLOs) of the EIS without having to directly manage the virtual infrastructure. TID and TP/UVA own the PIP-N and PIP-IT roles respectively, so that lower-LICL has been deployed in their facilities. TP acts also as a VIP and has deployed the upper-LICL. Finally, the VIO role is carried out by University of Amsterdam (UvA) which installed the SML. 


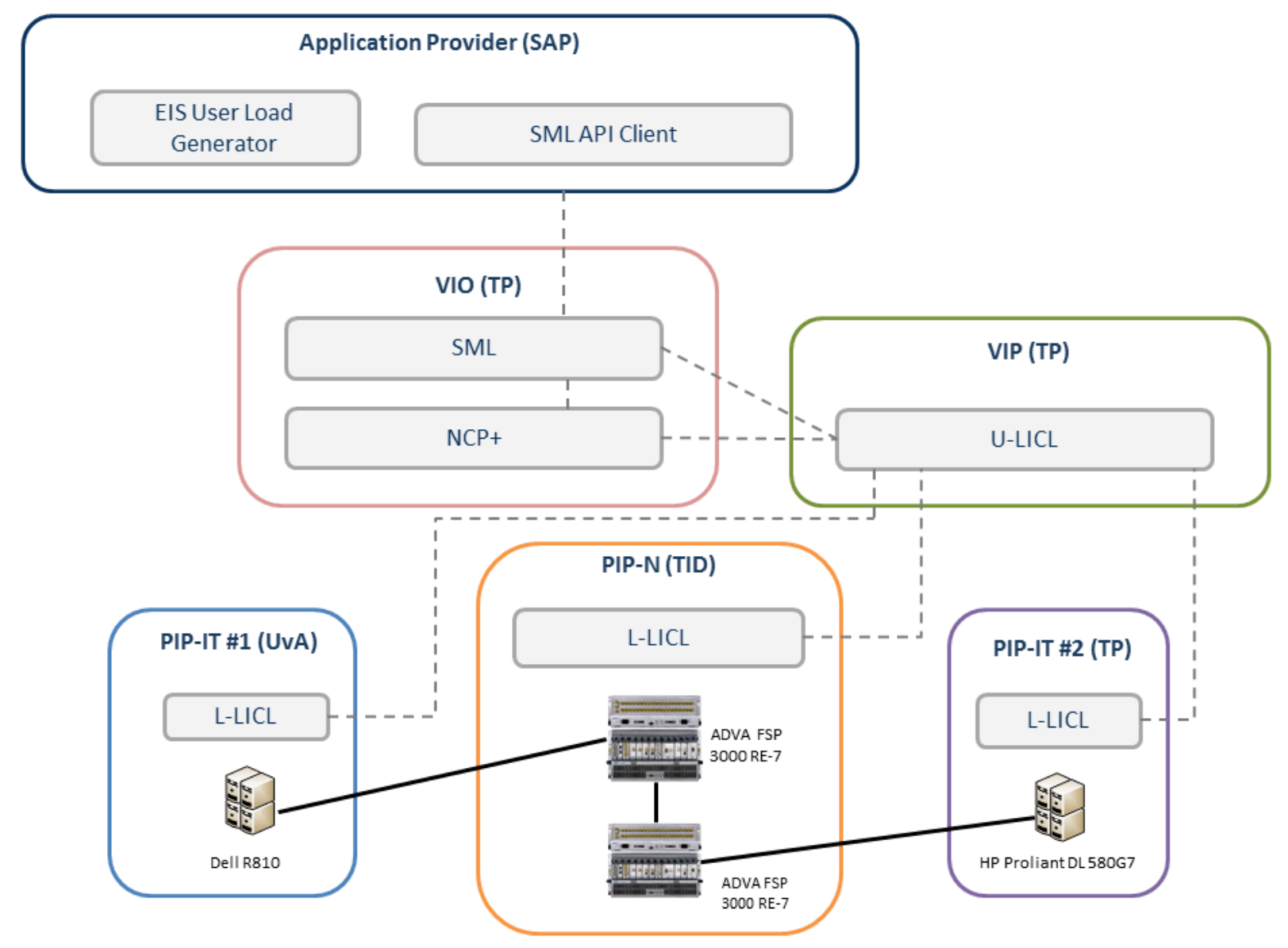

Figure 10: Resources and roles in Demonstrator 3.

The experiment is repeated for a number of iterations in three different configurations of the infrastructure. The first configuration is used to create a baseline with reliance on best-effort network and static configuration of infrastructure where there is no variation with load. The second configuration shows over-provisioned resources based on a calculation of the maximum set of resources that would be required. The load should hence never exceed the available resources, although there is excess available during periods of low load. Finally, the third configuration scales the virtual infrastructure resources according to a selected scaling algorithm.

\subsection{Demonstrator 4: Advanced Virtual Infrastructure and Service Management - Dynamic Re-planning}

In GEYSERS, the resources of the virtual infrastructure can be dynamically acquired and released through a set of mechanisms exposed by the LICL. This feature is fully exploited by the enhanced NCP, which is able to analyze the current network utilization and re-plan the underlying infrastructure by invoking a dynamic re-planning mechanism provided by the LICL during the operational phases. The virtual resources can be up- or downgraded according to the current traffic load and the service requests that are expected in the immediate future, allowing the operators to efficiently guarantee the network performance required by the active IT services and, at the same time, optimizing the resource utilization over 
the medium and long term. The VIP provides the dynamic virtual infrastructure re-planning service. The VIO, which is the client of this service, is in charge of requesting any modification of the rented infrastructure at any time. This capability allows all involved actors to apply the pay-as-you-grow model, allowing the virtual operator to avoid large initial investments (by requesting a VI with a limited size) and progressively rent additional resources to cope with growing business.

Demonstrator 4 depends on two main GEYSERS architecture components: upper-LICL and NCP+. VIP deploys the upper-LICL to provide virtual infrastructure management capabilities. The virtual network replanning functionalities are offered by upper-LICL software in a form of web service and are consumed by the $\mathrm{NCP}+$. The $\mathrm{NCP}+$ allows fully dynamic or manual triggering of re-planning actions as well as adaptation to dynamic infrastructure changes. NCP+ is deployed by the VIO.

The Demonstrator 4 evaluates and compares manual and dynamic methods for the VI re-planning, by providing usage examples for both of them. The dynamic re-planning is triggered automatically by the $\mathrm{NCP}+$, which contains internal logic for analysing overall network utilization. On the other hand, manual re-planning is triggered by the VIO network administrator, who would like to make required modifications to an already deployed rented virtual infrastructure. It must be noted that re-planning can be triggered manually but it is typically assumed to be performed fully automatically by the upper-LICL software.

Figure 11 presents the organizational structure of this demonstrator and roles performed by organizations participating in the Demonstrator 4. Additionally, GEYSERS software elements installed within each of the organizations and physical infrastructure are present (hardware equipment and data links).

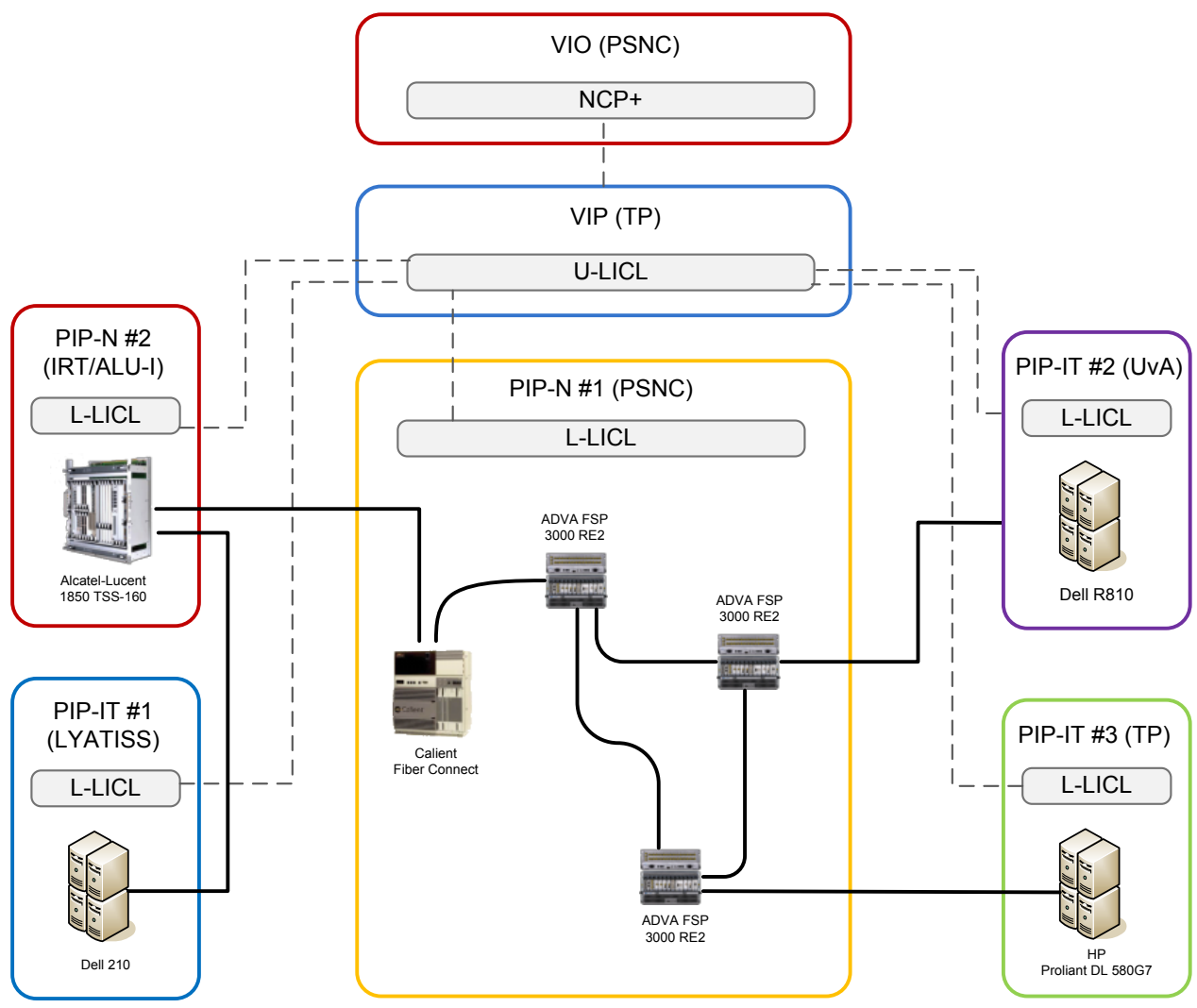

Figure 11: Resources and roles in Demonstrator 4. 
As depicted in Figure 11, physical resources installed for the Demonstrator 4 are provided by five independent organizations participating in the GEYSERS project. All these organizations perform the role of Physical Infrastructure Providers (PIPs) and deploy the lower-LICL software. Two of them are managing network infrastructure as the PIP-N. The rest are managing IT infrastructure as PIP-IT. Using the lower-LICL software they offer a part of managed physical infrastructure to the Virtual Infrastructure Provider (VIP). In this Demonstrator, TP is performing the role of VIP and deploys the upper-LICL software, and it is responsible for creating a single virtual infrastructure composed of logical resources offered by all five PIPs. Finally, PSNC is using a given virtual infrastructure as VIO and deploys an enhanced Network Control Plane (NCP+).

\section{Conclusions}

The GEYSERS project provides a novel approach to provisioning virtual infrastructures for the cloud based applications and services that may in general include multiple providers with heterogeneous service platforms distributed over multiple geographical locations and independent administrative domains. By introducing a minimal processing overhead and a completely new service model, a majority of new possibilities becomes available. These include, but are not limited to:

- Faster service provisioning - the process of cloud infrastructure establishment is a real on-demand service and can be achieved within minutes. Users do not have to deal with multiple network and IT providers independently. Instead, users receive a simple and straightforward interface in order to jointly provision both network and IT resources.

- Offered services better fit needs - the GEYSERS architecture can establish services based on requirements, assuring sufficient quality of experience while minimizing resource usage.

- Services can be dynamically adjusted - any time during operation of services the Virtual Infrastructure can be re-planned to follow variable user requirements.

- The GEYSERS architecture offers not just network connectivity, but an entirely independent and adaptive optical infrastructure set-up just for end-user tasks without requiring consumers to purchase and deploy any equipment on their own.

The testbed deployed by all involved partners closely mimics the typical scenario with inter-cloud multiprovider heterogeneous infrastructure services integration, which requires dedicated network infrastructure provisioning optimised for the given resources availability, distribution and ownership. First, the whole test network is owned by multiple independent parties (i.e. different project partners). This fact is very important from the business point of view because resource ownership boundaries become highly problematic to users desiring connectivity between two geographically distant locations. Moreover, the testbed spans the whole Europe continent, and as such very closely simulates all the potential transmission problems. Additionally, the GEYSERS testbed uses equipment manufactured by a variety of vendors and therefore gives great opportunity to perform interoperability tests among heterogeneous devices.

The current GEYSERS testbed and validation scenarios have been influenced by several factors. First of all, the novel concept proposed by GEYSERS needs to be well introduced to the wider network and IT community to gain greater interest, which can potentially result in commercial deployments in the future. A second factor is the need to validate the overall concept in practice in order to identify any potential concept weaknesses. Moreover, the GEYSERS infrastructure relies on several components such as the 
LICL, SML and NCP+, which either are extensions to existing protocols or entirely new elements that also need to be validated. The scope of GEYSERS demonstrators has been especially chosen to ease the testing process and allow verification of abovementioned components in a step-by-step manner. All demonstrators have been designed to demonstrate the role of each introduced layer within the GEYSERS architecture, to target different business and research communities.

The four demonstrators described in this paper have been executed in a testing environment in order to validate the GEYSERS architecture in real, networking conditions. The experiments have been executed following a detailed test plan prepared in a form of test cards, to verify a set of features of each GEYSERS component developed within the project.

The future operation of the testbed will provide an experimental platform for developing Intercloud Control and Management Plane and Intercloud Operational Framework [29]. It will also be valid for experimenting with the new dynamic trust management and scalable access control policy deployment initially proposed in the GEYSERS project [30]. Further research is being conducted on the SLA based infrastructure resources provisioning following the initial results presented in [27].

\section{Acknowledgements}

The work described in this paper has been partially founded by the GEYSERS project (http://www.geysers.eu), funded by the European Commission's Seventh Framework Programme (FP7/2007-2013) under grant agreement n. 248657.

\section{References}

[1] NIST SP 800-145, “A NIST definition of cloud computing", [online] Available: http://csrc.nist.gov/publications/nistpubs/800-145/SP800-145.pdf, September 2011

[2] NIST SP 500-292, Cloud Computing Reference Architecture, v1.0. [Online] http://collaborate.nist.gov/twiki-cloudcomputing/pub/CloudComputing/ReferenceArchitectureTaxonomy/NIST_SP_500-292_-090611.pdf September, 2011

[3] Baun, C., Kunze, M., Nimis, J., Tai. S., Cloud Computing: Web-Based Dynamic IT Services. ISBN 978-3-642-20916-1. Springer-Verlag Berlin Heidelberg, 2011

[4] Hao, F., Lakshman, T. V., Mukherjee, S., Song, H., Enhancing dynamic cloud-based services using network virtualization. Computer Communication Review 40(1): 67-74 (2010)

[5] Luis M. Contreras, Víctor López, Óscar González, Alejandro Tovar, Fernando Muñoz, Amanda Azañón, Juan P. Fernández-Palacios, Jesús Folgueira, "Towards Cloud-Ready Transport Networks", IEEE Communication Magazine, vol . 50, no. 9, pp. 48-55, September 2012

[6] C. Develder, M. De Leenheer, B. Dhoedt, M. Pickavet, D. Colle, F. De Turck and P. Demeester, "Optical networks for grid and cloud computing applications", Proc. IEEE, Vol. 100, No. 5, May 2012, pp. 1149-1167. doi:10.1109/JPROC.2011.2179629

[7] Q. Zhang, L. Cheng, and R. Boutaba, "Cloud computing: state-of-the-art and research challenges", Internet Serv Appl, vol. 1, no. 1, pp. 7-18, May 2010. 
[8] A. J. Ferrer, F. Hernández, J. Tordsson, E. Elmroth, A. Ali-Eldin, C. Zsigri, R. Sirvent, J. Guitart, R. M. Badia, K. Djemame, W. Ziegler, T. Dimitrakos, S. K. Nair, G. Kousiouris, K. Konstanteli, T. Varvarigou, B. Hudzia, A. Kipp, S. Wesner, M. Corrales, N. Forgó, T. Sharif, and C. Sheridan, "OPTIMIS: A holistic approach to cloud service provisioning," Future Generation Computer Systems, vol. 28, no. 1, pp. 66-77, Jan. 2012

[9] Cisco Global Cloud Index: Forecast and Methodology, 2010-2015, available at http://www.cisco.com/en/US/solutions/collateral/ns341/ns525/ns537/ns705/ns1175/Cloud_Index White Paper.pdf

[10] Cloud Reference Framework. Internet Draft, 28 June 2012, by B. Khasnabish, J. Chu, S. Ma, Y. Meng, N. So, P. Unbehagen, M. Morrow, M. Hasan, Y.Demchenko. [online] http://tools.ietf.org/html/draft-khasnabish-cloud-reference-framework-05.txt

[11] Demchenko, Y., C.Ngo, M.Makkes, R.Strijkers, C. de Laat, Intercloud Architecture for Interoperability and Integration. Proc. The 4th IEEE Conf. on Cloud Computing Technologies and Science (CloudCom2012), 3 - 6 December 2012, Taipei, Taiwan. IEEE Catalog Number: CFP12CLU-USB. ISBN: 978-1-4673-4509-5

[12] J.A. García-Espín, J. Ferrer Riera, S. Figuerola, M. Ghijsen, Y. Demchemko, J. Buysse, M. De Leenheer, C. Develder, F. Anhalt and S. Soudan, "Logical Infrastructure Composition Layer, the GEYSERS holistic approach for infrastructure virtualisation", in Proc. TERENA Networking Conference (TNC 2012), Reykjavík, Iceland, 21-24 May 2012

[13] J. Buysse, K. Georgakilas, A. Tzanakaki, M. De Leenheer, B. Dhoedt and C. Develder, "Energyefficient resource provisioning algorithms for optical clouds", IEEE/OSA J. Opt. Commun. Netw., Vol. 5, No. 3, Mar 2013, pp. 226-239. doi:10.1364/JOCN.5.000226

[14] GEYSERS Deliverable 4.1: GMPLS+/PCE+ Control Plane Architecture. Available at: http://www.geysers.eu/images/stories/deliverables/geysers-deliverable 4.1.pdf, November 2010

[15] GEYSERS Deliverable 4.2: NIPS user-network interface and procedures. Available at: http://www.geysers.eu/images/stories/deliverables/geysers-deliverable 4.2.pdf, November 2010

[16] G. Landi, N. Ciulli, J. Buysse, K. Georgakilas, M. Anastasopoulos, A. Tzanakaki, C. Develder, E. Escalona, D. Parniewicz, A. Binczewski, B. Belter, "A Network Control Plane architecture for ondemand co-provisioning of optical network and IT services", in Proc. Future Network Mobile Summit (FNMS 2012), Berlin, Germany, 4-6 Jul. 2012

[17] GEYSERS Deliverable D2.6 - Refined GEYSERS architecture, interface specification and service provisioning workflow. Available at: http://www.geysers.eu/images/stories/D2.6-final.pdf, January 2012

[18] GEANT Project: Transforming the way Researches collaborate. http://www.geant.net/pages/home.aspx

[19] GEYSERS Deliverable D5.1 - $\quad$ Testbed implementation. Available at: http://www.geysers.eu/images/stories/deliverables/geysers-deliverable_5.1-v1.6_final.pdf, July 2011

[20] OpenFlow in Europe Linking Infrastructure and Applications. FP7 Project. http://www.fp7-ofelia.eu/ [21] GÉANT Plus Service http://www.geant.net/Services/ConnectivityServices/Pages/GEANTPlus.aspx

[22] "OpenNebula Key Features and Functionality". OpenNebula documentation, http://www.opennebula.org/documentation:features, Retrieved 13 October 2011

[23] CloudWeaver - Cloud Network Control, http://www.lyatiss.com/wpcontent/uploads/2012/05/Lyatiss-CloudWeaver-Brochure.pdf 
[24] IEEE 802.1q, "IEEE Standard for Local and metropolitan area networks--Media Access Control (MAC) Bridges and Virtual Bridged Local Area Networks, http://standards.ieee.org/findstds/standard/802.1Q-2011.html, August, 2011

[25] Future Network Mobile Summit 2012, Berlin 2012. http://www.futurenetworksummit.eu/2012/

[26] JP. Vasseur, JL. Le Roux, "Path Computation Element (PCE) Communication Protocol (PCEP)", IETF RFC 5440, March 2009

[27] A. F. Antonescu, P. Robinson, and T. Braun. "Dynamic topology orchestration for distributed cloudbased applications", in $2^{\text {nd }}$ IEEE Symposium Network Cloud Computing and Applications, London, UK, 2012.

[28] Bosch, P., A.Duminuco, F.Pianese,T.L.Wood, "Telco Clouds and Virtual Telco: Consolidation, Convergance, and Beyond", Proc. Symposium on Integrated Network Management, 2011 IFIP/IEEE, Dublin, Ireland, 23-27 May 2011.

[29] Demchenko, Y., M. Makkes, R.Strijkers, C.Ngo, C. de Laat, "Intercloud Architecture Framework for Heterogeneous Multi-Provider Cloud based Infrastructure Services Provisioning", The International Journal of Next-Generation Computing (IJNGC). July 2013.

[30] Ngo, C., Y.Demchenko, C. de Laat, Toward a Dynamic Trust Establishment Approach for Multiprovider Intercloud EnvironmentThe 4th IEEE Conf. on Cloud Computing Technologies and Science (CloudCom2012), 3 - 6 December 2012, Taipei, Taiwan. ISBN: 978-1-4673-4509-5

[31] M. Campanella, F. Farina "The FEDERICA Infrastructure and Experience", Computer Networks Special Issue on Future Internet Testbeds

[32] M. Berman, J.S. Chase, L. Landweber, A. Nakao, M. Ott, D. Raychaudhuri, R. Ricci, I. Seskar, "GENI: A federated Testbed for Innovative Network Experiments", Computer Networks Special Issue on Future Internet Testbeds

[33] M. Suñé, L. Bergesio, H. Woesner, T. Rothe, A. Köpsel, D. Colle, B. Puype, D. Simeonidou, R. Nejabati, M. Channegowda, M. Kind, T. Dietz, A. Autenrieth, V. Kotronis, E. Salvadori, S. Salsano, M. Körner, S. Sharma, "Design and Implementation of the OFELIA FP7 Facility: The European OpenFlow Testbed", Computer Networks Special Issue on Future Internet Testbeds 